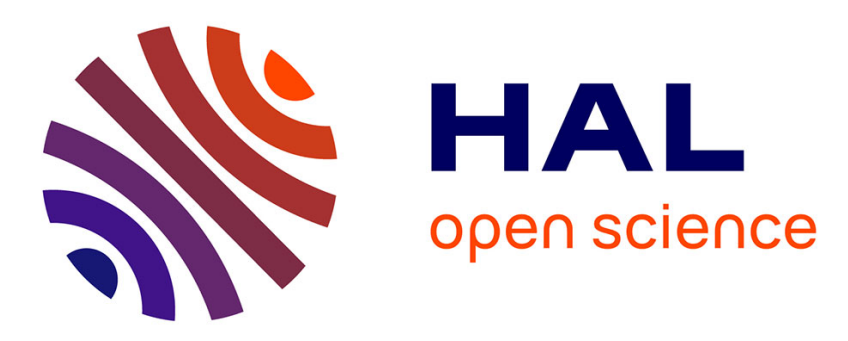

\title{
The RGM data structure: a nominal interpretation of an acquired high point density model for automatic tolerance inspection
}

\author{
Paolo Di Stefano, Luca Di Angelo, Annaeva Morabito
}

\section{To cite this version:}

Paolo Di Stefano, Luca Di Angelo, Annaeva Morabito. The RGM data structure: a nominal interpretation of an acquired high point density model for automatic tolerance inspection. International Journal of Production Research, 2011, pp.1. 10.1080/00207543.2011.579638 . hal-00726658

\section{HAL Id: hal-00726658 https://hal.science/hal-00726658}

Submitted on 31 Aug 2012

HAL is a multi-disciplinary open access archive for the deposit and dissemination of scientific research documents, whether they are published or not. The documents may come from teaching and research institutions in France or abroad, or from public or private research centers.
L'archive ouverte pluridisciplinaire HAL, est destinée au dépôt et à la diffusion de documents scientifiques de niveau recherche, publiés ou non, émanant des établissements d'enseignement et de recherche français ou étrangers, des laboratoires publics ou privés. 




The RGM data structure: a nominal interpretation of an acquired high point density model for automatic tolerance inspection

\begin{tabular}{|r|l|}
\hline Journal: & International Journal of Production Research \\
\hline Manuscript ID: & TPRS-2010-IJPR-1058.R1 \\
\hline Manuscript Type: & Original Manuscript \\
\hline Date Submitted by the \\
Author: & 20 -Jan-2011 \\
\hline Complete List of Authors: & $\begin{array}{l}\text { Di Stefano, Paolo; Universita' degli Studi di L'Aquila, Mechanical } \\
\text { Engineering } \\
\text { Di Angelo, Luca; Universita' degli Studi di L'Aquila, Mechanical } \\
\text { Engineering } \\
\text { Morabito, Annaeva; Universita' di Lecce, Ingegneria dell'innovazione }\end{array}$ \\
\hline Keywords: & TOLERANCING, AUTOMATED INSPECTION \\
\hline Keywords (user): & Three dimensional Metrology \\
\hline
\end{tabular}




\title{
The $R G M$ data structure: a nominal interpretation of an acquired high point density model for automatic tolerance inspection
}

\author{
L. DI ANGELO $\left({ }^{1}\right)$, P. DI STEFANO $\left({ }^{1}\right)$, A. E. MORABITO $\left({ }^{2}\right)$ \\ $\left({ }^{1}\right)$ Department of Industrial Engineering, University of L'Aquila, \\ Loc. Monteluco di Roio, 67030 L'Aquila, Italy \\ $\left(^{2}\right)$ Department of “Ingegneria dell'Innovazione”, University of Lecce, \\ Via per Monteroni, 73100 Lecce, Italy
}

(Received; final version received)

\begin{abstract}
In a previous paper (Di Angelo et al. 2010) we proposed an original methodology for the automation of the geometric inspection, starting from an acquired high-density surface. That approach performed a recognition process on the acquired data aiming at the identification of some Intrinsic Nominal References. An Intrinsic Nominal Reference was detected when a geometric property was recognised to be common to a set of adjacent points in the 3D data set representing the acquired object. The recognition of these properties was carried out based on some rules. Starting from these concepts, a new specification language was defined, which is based on recognisable geometric entities. This paper expands the category of Intrinsic Nominal References to include new mutual intrinsic orientation, location and dimensional properties pertaining to $3 \mathrm{D}$ features. This approach involves the automatic construction of a geometric reference model for a scanned workpiece, called Recognised Geometric Model (RGM). The domain of the representable entities within the RGM strictly depends on the rules used for the recognition of the intrinsic properties. In particular, this paper focuses on the rules for the recognition of the orientation and location properties between non-ideal features. When using the $R G M$, tolerances are specified according to the set of available and recognisable intrinsic nominal references. Based on the geometric product specification, the $R G M$ data structure can be queried to capture some quantitative information concerning special intrinsic geometric parameters and/or non-idealities.
\end{abstract}

Keywords: ISO Tolerancing, Three dimensional Metrology, Triangular Meshes, Shape recognition, Automated inspection

\section{Introduction}

Geometric inspection has actually become critical in the last few years due, on the one hand, to the increase in geometric complexity and product variety, and, on the other hand, to the evermore higher demands for geometric accuracy. Furthermore, and as far as some applications are concerned, such as, for instance, the aerospace engineering, each and every manufactured part, and not just a sample of them, needs inspecting. Consequently, a reliable, effective, and automated inspection system will improve the industrial manufacturing responsiveness so as to increase product competition. Most of these requirements (for example, the requirements for assemblability) can be expressed by using the geometric product specifications defined by the International Organization for Standardization (ISO) or by the ANSI/ASME. Some years ago, the ISO proposed a new language to express the geometric product specifications. This language, which is based on mathematical principles, is usually acronymed GPS (Geometric Product Specification).

Any language intended for tolerance specification must strictly be linked to the instruments available for the inspection. The GPS normative is mainly based on 
the inspection capabilities of CMM (Coordinates Measurement Machine), gauges, dial gauges, etc. The concepts of tolerance and dimension also depend on the scheme of representation adopted to specify the geometric entities. Currently, in the GPS standards, these concepts refer to a $2 \mathrm{D}$ representation obtained by projecting the objects orthogonally onto a plane.

Nowadays, with the advent of high-resolution optical digitisers, new prospects are offered for real automatic geometric inspection (Gao et al. 2006) and, by extension, for tolerance specification (Srinivasan, 2001). The measurement process carried out by these high-resolution digitisers allows of a 3D acquisition of the real object. Many data points taken from surfaces of the inspected object (with a resolution up to $10 \mu \mathrm{m}$ ) allow us to define both new tolerance categories and new procedures in order to verify the traditional ones.

The development of a new methodology for automatic tolerance inspection starting from an acquired high-density 3D model is an ongoing activity by our research group. To that end, the new approach carries out a recognition process on the acquired data which serves to identify some intrinsic nominal references (Di Angelo et al. 2010). Taking these references as a starting point, new and old categories of form error are defined and some procedures are proposed for a more robust verification of traditional tolerance categories. There exists an Intrinsic Nominal Reference when a geometric property is recognised to be common to a set of adjacent points from the 3D data set representing the acquired object.

In this paper, the category of intrinsic nominal references is widened to include new mutual intrinsic orientation, location and dimensional properties pertaining to $3 \mathrm{D}$ features. This approach involves the automatic construction of a geometric reference model for the scanned workpiece, called Recognised Geometric Model $(R G M)$. In order to evaluate the non-idealities ${ }^{l}$ of the acquired object, the unstructured $3 \mathrm{D}$ data points in the $R G M$ are clustered around some recognised nominal categories. The recognition of these categories is carried out based on some rules, which therefore play a leading role in the definition of the domain of the representable entities within the $R G M$. In particular, this paper focuses on the rules concerning the recognition of intrinsic properties in the orientation and location relationships between non-ideal features. Those rules intended for geometric form recognition have already been presented in (Di Angelo et. al. 2010).

\section{Literature review}

For the last few years, and as far as research is concerned, a considerable amount of effort has been directed toward the development of methodologies aiming at performing an effective and reliable automated geometric tolerance verification (Prieto et al. 2002, Li and Gu 2005, Gao et al. 2006, Wong et al. 2006). A great number of these methodologies require knowing the CAD model for the workpiece under inspection. The CAD model provides us with the nominal references in the form of parameterised equations describing the surfaces of the geometric model (Prieto et al. 2002, $\mathrm{Li}$ and $\mathrm{Gu}$ 2004, $\mathrm{Li}$ and $\mathrm{Gu}$ 2005, Gao et al. 2006) and it often contains an explicit coding of the orientation and location relationships between the surfaces. Since tolerance specifications usually refer to some features of the

\footnotetext{
${ }^{1}$ We prefer the term 'non-ideality' over 'error'. Whereas the error can be corrected, it is not possible to correct the non-ideality since it is inevitably associated with real objects to which the concept of perfection or ideality cannot be applied.
} 
workpiece, the mapping between one surface (or feature) of the CAD model and the corresponding scanned point sub-cloud needs to be performed. Tolerance specifications can be either included in the CAD model as textual information, or interactively defined by the user. However, no standard language capable of specifying tolerances and suited to automatic verification has yet been defined. Prieto et al. (2002) proposed and implemented a methodology for automated inspection of manufactured parts. Their methodology first registers the experimental point cloud with the corresponding CAD model of the workpiece by using the Iterative Closest Point (ICP) algorithm, and then segments the 3D point cloud by associating the points matching the same local geometric properties with the nearest CAD surface. This methodology is able to verify both dimensional and geometric tolerances.

In (Gao et al. 2006) the authors develop an automated geometric inspection system within a commercial RE (Reverse Engineering) software. A Nominal Inspection Frame (NIF) for a CAD model or a digitised reference model (Master Model) is defined for which every dimensional and geometric tolerance specification may be defined and interactively specified by the user. The GD\&T items, which can be defined by the $N I F$, pertain to:

- flatness;

- datum (point, line, axis or plane);

- parallelism;

- perpendicularity;

- true position.

Once the NIF is created, the scanned data are then aligned with the nominal model; GD\&T items on the measured parts are automatically computed and extracted from the NIF previously defined by means of the software functions and macro programs.

In (Wong et al. 2006), the authors develop an algorithmic approach for inspection feature recognition directly from a CAD model. Several types of inspection features (external, internal, offset and free form), usually found in industrial practice, can be extracted. In order to facilitate the extraction and recognition of an inspection feature, a multi-attribute spatial graph is proposed where both the nodes and the edges may have specified attributes. The node attributes specify the geometric type of a CAD face (planar, cylindrical or free form). The edge attributes identify some spatial relationships between pairs of adjacent and non-adjacent faces. This graph is capable of supporting the extraction of inspection features for linear as well as angular dimensional measurement. The syntax proposed by (Wong et al. 2006) for the formal representation of inspection features provides us with a basis for automating inspection process planning by using conventional metrological equipment (comparator, dial gauge, etc.). Some specific inspection equipment as well as a particular application method can be recommended for each dimensional inspection feature. Moreover, the boundary face list provides the corresponding constraints on the access, location, alignment and handling of the measuring face.

\section{The $R G M$ operations}

The Recognised Geometric Model (RGM) is a special kind of geometry representation of a measured object deriving from the recognition of some ideal properties of the object. Figure 1 shows the flowchart for the process of derivation and query of the $R G M$. This representation is drawn from a high density point cloud, which reproduces the real object being acquired. The recognition of these properties 
(form, orientation and location) is carried out based on some rules, which play a leading role in the $R G M$ construction.

Figure 1: Flowchart for the process of derivation and query of the $R G M$

\subsection{The non-ideal feature segmentation}

$R G M$ construction involves a complex segmentation process directed toward the identification of the non-ideal features and the associated category of recognisable ideal properties. We define a non-ideal feature as a set of adjacent points that are recognised to be smooth, of the same type (flat, umbilical, ruled and generic) and to pertain to a unique regular surface. The type of point is deduced by evaluating some differential geometric properties. This definition completes the vagueness of the ISO standard (ISO/TS 17450-1: 2005) which defines the non-ideal feature as the "imperfect feature fully dependent on the non-ideal surface model (skin model)". The ISO definition seems to refer to a property of a non-ideal feature (the imperfection) rather than to its description. According to the concept of non-ideal feature, the acquired data in the RGM are segmented in a way that is consistent with recognisable patches of the object's surface. The set of non-ideal features represents the object with the exception of its non-regular parts (ridges and singularities). These nonregular parts do not follow a recognisable rule and are therefore excluded from further elaborations in $R G M$ (Di Angelo and Di Stefano, 2010). This work makes use of a surface hybrid segmentation approach (Di Angelo et al. 2007), based on fuzzy logic. The non ideal feature segmentation gives results in any case. In the case of an object embodied by one and only free form surface, the feature segmentation returns an only entity.

\subsection{The ideal feature recognition}

According to GPS standards, the feature operation called association makes it possible "to fit an ideal feature to a non ideal feature based on a criterion" (ISO/TS 17450-1: 2005). These criteria (herein referred to as recognition rules) are based on the evaluation of some local and global differential properties of the segmented model. The present work classifies the ideal features into two main categories: analytical and non-analytical features. Analytical features are those recognised to pertain to an analytical geometric surface (plane, sphere, cylinder, cone, etc.). For these ideal features, an analytical type of geometric surface is automatically recognised and associated with the non-ideal feature. Those ideal features which are not analytical are defined to be non-analytical features. Non-analytical features also include surfaces characterised by some specific and recognisable geometric properties. Based on the recurrence of specific differential geometric properties among the points pertaining to the non-ideal feature, they can be classified as: generic extruded, generic cone, generic axially-symmetric. Henceforth we will be referring to them as generic ruled $(G R)$ and generic axially-symmetric $(G A)$. The non-analytical features which cannot be included within any of the previous categories are free form features. In any case, for any non-analytical feature a parameterised equation can be associated with a CAD model by a registration process. In the $R G M$ data structure the concept of ideal feature has to do with the qualitative property (or attribute) of a 


\section{Table 1. The Intrinsic Characteristics of the ISRs}

For a non-analytical feature the shape reference must be given explicitly. In some special types of non-ideal features, such as GR and GA, only IDRs and ILRs can be detected. An $I D R$ is an analytically-known geometric entity deriving from the evaluation of some geometric properties of a feature. Generally speaking, these entities are not physical; for this reason, they are not directly measurable on the object, but can nevertheless be derived from the measured surface. Examples of IDRs are the axis and the circular sections of axially-symmetric surfaces. Whereas the circular sections are physical entities, the axis is not. In many cases these references are the situation features of the non-ideal features, since they serve as references for feature location and/or orientation. The situation features for each type of non-ideal feature here considered are reported in table 2. They are ideal features of the following types: point $(\wp)$, straight line $(r)$ or plane $(\Pi)$.

Table 2. The situation features for each type of non-ideal feature here considered

Other IDRs could be defined based on functional or manufacturing properties of the acquired object. For the gears shown in figure 2, the base cylinder or cone could represent the Intrinsic Derived References of the object and their axes could represent the location characteristic of the gear. A further example could be the symmetry plane of a free-form mirrored surface (figure $2 \mathrm{c}$ ). The implementation of these IDRs in the RGM requires the introduction of specific recognition rules. 
Both shape and derived references are estimated by approximating the point cloud associated with a non-ideal feature through one or more association operations. Depending on the association rule which is used, different intrinsic references can be estimated.

The Intrinsic Local Reference (ILR) is an original type of intrinsic nominal reference since it is not considered in the current tolerancing standards. It deals with the uniformity of some intrinsic differential geometric properties, such as: regularity, curvature recurrence, and so on. These references do not pertain to the global analytical properties of a surface, but rather to properties which characterise it locally. In the $R G M$ two ILRs are introduced. The first one is the regularity of the points pertaining to a non-ideal feature. Surface regularity locally measures the membership of each point to a regular surface which approximates its neighbourhood. Another ILR concerns the ruled surfaces (Di Angelo et al. 2010). This reference has to do with the recognition of the ruled property of a non-ideal feature. This kind of intrinsic reference can be recognised for cylinder, cone and $G R$.

Figure 2: Examples of IDRs: a) the base cylinder for a cylindrical gear, b) the base cone for a conical gear and c) the symmetry plane for a free-form specular surface.

Table 3 shows the $I S R \mathrm{~s}$, the IDRs and the ILRs, related to several ideal feature types.

The types of ideal feature which can be included within the $R G M$ strictly depend on the capability of said software to recognise them. In fact, table 3 only reports those types of ideal features that the prototypal software here implemented can recognise. However, further categories of analytical and non-analytical features can be added to the $R G M$.

Other intrinsic properties can be recognised in the mutual geometric relationships between non-ideal features. These relationships can be subdivided into: orientation properties and location properties. Each of them has a corresponding nominal category with which an intrinsic reference (a specific orientation or location) can be associated. Both Intrinsic Orientation and Intrinsic Location References can be identified between non-ideal features which are located based on their own situation features. The orientation relationship is a property which can be evaluated between directions. The Intrinsic Orientation Reference (IOR) refers to the mutual geometric properties of parallelism and perpendicularity between non-ideal features. Furthermore, some angular values can be recognised to be very close to angular values which frequently occur in mechanical workpieces, such as $30^{\circ}, 45^{\circ}$ and $60^{\circ}$. The Intrinsic Location Reference (ILoR) includes special types of location relationships. They are: concentricity, coaxiality and coincidence. The recognition of these intrinsic mutual location relationships requires that a localised situation feature should be identified for each non-ideal feature. A situation feature is said to be localised if it is possible to unequivocally identify its location within an arbitrarily given reference frame. For example, the axis of a $G A$ localises the surface to a plane which is orthogonal to the axis. On the contrary, the extrusion direction, that is to say, the situation feature recognised for a generic extruded surface, is not a localised situation feature. Therefore, for this kind of surface it is only possible to recognise orientation properties and not location relationships. 
For the recognition of the orientation and location properties it seems more suitable to classify the features into planar features (henceforth referred to as $P$ feature) and features whose situation feature is an axis or an extrusion direction (henceforth referred to as $R$-feature).

\subsection{Dimensionable intrinsic parameters' evaluation}

Within the $R G M$, dimensionable geometric parameters are both the intrinsic characteristics and the intrinsic situation characteristics. According to the definition given by the GPS standards, situation parameters describe "the relative situation (location or orientation) between two situation features" (ISO/TS 17450-1: 2005). They can be further divided into location parameters and orientation parameters. The former are expressed by length (distance) values; the latter are expressed by angular values. In $R G M$ an intrinsic location characteristic is automatically associated whenever an intrinsic reference, either analytical or derived, is recognised to be parallel to another reference. The dimensionability of the parallelism is therefore an intrinsic reference. This dimensionable characteristic does not identify an intrinsic reference value for the dimension, but a reference dimension can nevertheless be specified for it. This operation is here referred to as dimensional registration.

\section{The $R G M$ data structure}

$R G M$ is an idealised representation of an acquired real object. This geometric representation can be described by means of a hypergraph structure denoted by $R G M(\mathrm{~V}, \varepsilon)$, where $\mathrm{V}$ is the finite set of nodes $\mathrm{v}_{\mathrm{i}}$ of the hypergraph. Each node is associated with a non-ideal feature of the measured object. Some labels are assigned to each node of the hypergraph, which describe the non-ideal feature attributes (type of ideal feature, intrinsic characteristics, situation features, $R$-feature or $P$-feature). In $\operatorname{RGM}(\mathrm{V}, \varepsilon), \varepsilon$ is a family of two sub-sets of $\mathrm{V}\left(\varepsilon=\left\{\mathrm{V}_{\mathrm{A}}, \mathrm{V}_{\mathrm{GR}}\right\}\right)$ which are known as hyper-edges and which respectively represent the sets of non-ideal features for which adjacency relationships and mutual geometric properties are respectively recognised. Whereas the adjacency relationships are recognised between non-ideal features, the mutual geometric relationships are recognised between the situation features of the non-ideal features.

In RGM both the Adjacency Graph (AG) and the Mutual Geometric Relationship Hypergraph (MGRH) can be identified. In the $\mathrm{AG}=\left(\mathrm{V}_{\mathrm{A}}, \mathrm{E}_{\mathrm{A}}\right)$, an arc $\mathrm{e}_{\mathrm{i}}$ $\in \mathrm{E}_{\mathrm{A}}$ represents an adjacency between neighbouring non-ideal features. For every pair of adjacent non-ideal features there exists unique sole arc $\mathrm{e}_{\mathrm{i}}$ connecting the corresponding nodes.

In the hypergraph MGRH $=\left(\mathrm{V}_{\mathrm{GR}}, \varepsilon_{\mathrm{GR}}\right), \varepsilon_{\mathrm{GR}}$ is a family of two hyper-edges $\left(\varepsilon_{\mathrm{GR}}=\left\{\mathrm{V}_{\mathrm{OR}}, \mathrm{V}_{\mathrm{LR}}\right\}\right) . \mathrm{V}_{\mathrm{OR}}$ is the set of non-ideal features for which intrinsic orientation properties are recognised. $V_{L R}$ in turn represents the set of non-ideal features for which intrinsic location properties are detected. Taking the MGRH as a starting point, several graphs can be automatically derived to represent each type of orientation and location property recognised between the non-ideal features. These graphs are defined in the following sections. 


\section{The recognition of the intrinsic orientation properties}

Some intrinsic orientation properties can be recognised between non-ideal features. They are parallelism, perpendicularity and special angular orientations (such as, for example, $30^{\circ}, 45^{\circ}$ and $60^{\circ}$ ). These properties are recognisable between non ideal features whose situation features include a spatial direction, such as plane, cylinder, cone, $G A$ and generic extruded (table 2).

In the $R G M$ a set of $\mathrm{N}$ parallel features of the same type constitutes a system of homogeneous parallel entities $\left(S_{h / /}\right)$. Two types of systems can be identified: the system including $R$ - features, which is denoted by $\mathrm{S}_{/ / \mathrm{R}}$, and that including $P$


analysing the Mutual Parallelism Relationship Graph for $R-$ features $\mathrm{G}_{/ / \mathrm{R}}=\left(\mathrm{V}_{/ / \mathrm{R}}\right.$, $\left.\mathrm{E}_{/ / \mathrm{R}}\right)$ and the Mutual Parallelism Relationship Graph for $P-$ features $\mathrm{G}_{/ / \mathrm{P}}=\left(\mathrm{V}_{/ / \mathrm{P}}\right.$, $\left.\mathrm{E}_{/ / \mathrm{P}}\right)$, respectively. $\mathrm{G}_{/ / \mathrm{R}}\left(\mathrm{G}_{/ / \mathrm{P}}\right)$ is a graph where each node represents an $R$-feature ( $P$ - feature) and each edge represents a mutual parallelism property recognised between $R$-features $\left(P\right.$-features). In $\mathrm{G}_{/ / \mathrm{R}}\left(\mathrm{G}_{/ / \mathrm{P}}\right)$ there are as many components (i.e. maximal connected sub-graphs (Gross et al. 2004)) as spatial directions for which a parallelism property has been recognised. A component of $\mathrm{G}_{/ / \mathrm{R}}\left(\mathrm{G}_{/ / \mathrm{P}}\right)$, characterised by $\mathrm{N}$ nodes, is a system of homogeneous parallel entities $S_{/ / R}\left(\mathrm{~S}_{/ / \mathrm{P}}\right)$ if the degree of each node is equal to $\mathrm{N}-1$. For example, for the object shown in figure $3 \mathrm{a}$, four $S_{h / /}$ systems are recognised (figure $3 \mathrm{~b}$ ). Each $S_{h / /}$ in $\mathrm{G}_{/ / \mathrm{P}}$ or $\mathrm{G}_{/ / \mathrm{R}}$ is associated with a reference spatial direction.

In order to recognise an intrinsic orientation property between non-ideal features, some rules must be firstly defined. In this work, the rules to recognise these properties are based on the evaluation of the dot product between the spatial directions of the ideal features. These rules vary depending on whether the features are of the same type ( $P$-features or $R$-features) or of a different type (see table 4 ). Due to the non-ideality of real objects, the dot product never exactly matches the ideal value. Consequently, the recognition of the orientation property cannot be deduced from the mathematical verification of an equality. In this paper, however, the values are considered to 'match' if the dot product value falls within a properly given tolerated range around the ideal value (reported in table 4 for the various properties). A further control consists in verifying the coherence of the parallelism relationships when these are recognised in pairs between $\mathrm{N}$ features $(\mathrm{N} \geq 3)$ of the same type $(R-$ features or $P$-features). In other words, the transitive property must be verified. For instance, for $\mathrm{N}=3$ the transitive property can be enunciated in the following way:

iffeature $A / /$ feature $B$ and feature $B / /$ feature $C \rightarrow$ feature $A / /$ feature $C$

Table 4. The rules to recognise the intrinsic orientation properties

Figure 3: An example of recognition of orientation properties and related graphs

The transitive property, in practical cases, cannot be verified and ambiguities arise due to errors of type I and errors of type II. An error of type I occurs when the orientation property is true but nonetheless fails to be recognised. An error of type II 
Figure 4: Derivation of several systems of homogenous parallel entities from one system that is not $S_{h / /}$

The transitive property for parallelism (1) cannot be verified between different types of non-ideal features. For instance, if $\mathrm{A}$ is an $R$-feature, B is a $P$-feature, and $\mathrm{C}$ is an $R$-feature, the above property is not necessarily satisfied for any directions of $R$-features. For a given $P$-feature there could be $R$-features which are parallel to it, but not parallel to each other. For the same reason, the transitive property is not verified between $P$-features which are all parallel to the same $R$-feature.

\subsection{The Mutual Orientation Relationship Graph (MORG)}

The concept of system of homogeneous parallel entities is functional to build the Mutual Orientation Relationship Graph $M O R G=\left(\mathrm{V}_{\mathrm{OR}}, \mathrm{E}_{\mathrm{OR}}\right)$. The nodes of $M O R G\left(\mathrm{~V}_{\mathrm{OR}}\right)$ are systems of homogeneous parallel entities $S_{h / /}$ or single features which do not belong to any $S_{h / /}$. The arcs $\left(\mathrm{E}_{\mathrm{OR}}\right)$ identify mutual orientation properties between connected nodes. A label is assigned to each edge which specifies the type of recognised orientation (parallelism, perpendicularity, $30^{\circ}, 45^{\circ}$ and $60^{\circ}$ ). Figure $3 \mathrm{c}$ illustrates an example of $M O R G$. It refers to the object shown in figure $3 \mathrm{a}$.

Thanks to the concept of system of homogeneous parallel entities, if an orientation property is recognised between one feature of a system $S_{h / 1}$ and one feature of a system $S_{h / / 2}$, then it is possible to deduce the satisfaction of this property between each feature of $S_{h / / 1}$ and each feature of $S_{h / 2}$.

\section{The recognition of the Intrinsic Location Characteristic}

For each parallelism property which is recognised between two localised situation features, there can be a dimensional parameter referred to as intrinsic location characteristic. This parameter represents the distance between two parallel ideal entities approximating the two situation features recognised to be parallel to each other. For example, for the system $S_{/ / P}$ of three planar features shown in figure 5, three intrinsic location characteristics can be identified. In the same figure, $d_{A-B}$ is the distance between the parallel ideal planes $\Pi_{\mathrm{A} / / \mathrm{A}-\mathrm{B}}$ and $\Pi_{\mathrm{B} / / \mathrm{A}-\mathrm{B}}$ respectively approximating the non-ideal features $\mathrm{P}_{\mathrm{A}}$ and $\mathrm{P}_{\mathrm{B}}$, which are recognised to be parallel to 
each other. This pair of ideally parallel features will be referred to as "virtual gauge", working analogously to a physical gauge, which matches the features to be measured with the two parallel surfaces.

In an ideal model, the coherence between the distances of three or more situation features must be verified in order to satisfy the non-contradiction principle so that: $\mathrm{d}_{\mathrm{A}-\mathrm{B}}+\mathrm{d}_{\mathrm{B}-\mathrm{C}}=\mathrm{d}_{\mathrm{A}-\mathrm{C}}$. On the contrary, in an acquired object in which the features are recognised to be parallel despite the fact that they are not really parallel to each other, every dimension can be evaluated independently from the others. For this reason, $\mathrm{d}_{\mathrm{A}-\mathrm{B}}+\mathrm{d}_{\mathrm{B}-\mathrm{C}} \neq \mathrm{d}_{\mathrm{A}-\mathrm{C}}$ and the representation of the three distances are not really a redundancy.

In the $R G M$ query, several values for the Intrinsic Location parameter can be obtained depending on the association rule used for approximating the two non-ideal features (for example, the least squares fitting $\mathrm{L}_{2}$, the upper or inner envelope fitting, etc.).

In order to represent the intrinsic location characteristics in the RGM, a specific graph is built which is referred to as Intrinsic Location Parameters Graph $(I L P G)$. It is defined further down in this paper, in section 7.1.

Figure 5: The three intrinsic location characteristics identified for a system $S_{/ / P}$ of three planar features

\section{The recognition of the intrinsic location properties}

Other intrinsic location properties are: coaxiality, concentricity and coincidence. Table 5 shows the intrinsic location properties which are here recognised. In order to detect them some recognition rules must be firstly defined.

Table 5. The intrinsic location properties here recognised for the several feature types

Coaxiality is the property of two ideal axially-symmetric features having the same axis. Some recognition rules need to be defined for the recognition of this property between non-ideal features. The rule which is here being followed involves first the parallelism recognition and then the evaluation of the related intrinsic location characteristic (representing the distance between the axes of the features). Coaxiality is recognised if the distance value (estimated by approximating the axialsymmetric features by the $\mathrm{L}_{2}$-rule) falls within a properly given tolerated range around 0 .

In the GPS standards, the concept of concentricity is defined between features projected onto a plane (ISO 1101: 2004). In this work this concept is extended to include 3D features. Concentricity is then the property of two spherical ideal features having the same centre. As far as the rule for the recognition of this property is concerned, it should be based on the comparison of the distance between the centres of the two spheres (analytically identified by the $\mathrm{L}_{2}$-rule) with a properly given tolerated value.

Coincidence is the property of two features which are one on the prosecution of the other. Some recognition rules are also defined in order to detect this property 
Table 6 . The coincidence property recognition

When intrinsic location properties of a specific type (concentricity, coaxiality or coincidence) are recognised in pairs between $\mathrm{N}$ features $(\mathrm{N} \geq 3)$, the transitive property must be verified. Any set of $\mathrm{N}$ features for which $\sum_{\mathrm{i}=1}^{\mathrm{N}}(\mathrm{N}-\mathrm{i})$ location properties are recognised in pairs is said to automatically satisfy the coherence imposed by the transitive property. This set of features is referred to as system of coherent localised entities $\left(S_{L o}\right)$.

In order to represent intrinsic location properties and identify the different types of system of coherent localised entities, a specific graph is defined. The mutual location relationship graph $M L R G=\left(\mathrm{V}_{\mathrm{LR}}, \mathrm{E}_{\mathrm{LR}}\right)$ is a graph where each node identifies mutual location properties between connected nodes. Each edge is assigned a label which reports the type of location recognised (coaxiality, concentricity and coincidence).

The systems of coherent localised entities can be automatically detected by analysing the above-mentioned graph. In the graph there are as many components as non-ideal features for which an intrinsic location property has been recognised. A component, characterised by $\mathrm{N}$ nodes, represents a system of coherent localised entities $S_{L o}$ (for a specific type of location property) if the degree of each node is equal to $\mathrm{N}-1$. If the degree of any nodes of the component is less than $\mathrm{N}-1$, the coherence verification, imposed by the need to satisfy the transitive property, cannot be possibly carried out. The subsequent incoherence could be solved either by imposing the missing location conditions or by splitting the component into two or more systems of coherent localised entities $S_{L o}$. The first approach could give rise to errors of type II, whereas the second one could generate errors of type I.

A reference situation feature is associated with each $S_{L o}$. This turns out to be useful during the phase of RGM query whenever the user asks for the evaluation of the intrinsic situation characteristics between two non-ideal features. If one or both non-ideal features belong to a system of coherent localised entities $S_{L o}$, it is possible to consider the reference situation feature (associated with $S_{L o}$ ) rather than the situation feature of the single feature. This allows us to obtain a value for the situation characteristic which is coherent with satisfying the transitive property. 


\subsection{Intrinsic Location Parameters Graph (ILPG)}

Thanks to the concept of system of coherent localised entities, the Intrinsic Location Parameters Graph (ILPG $=\left(V_{L P}, E_{L P}\right)$ ), which has been introduced in section 6, can be efficiently defined. This graph is the data structure used for describing, within the RGM, the intrinsic location characteristics identified in the acquired object.

The nodes of ILPG are systems of coherent localised entities $S_{L o}$ or single features which do not belong to any $S_{L o}$. In either case the features can be planar or axial-symmetric since the intrinsic location characteristic can only be defined (see section 6) for these types. The arcs of ILPG represent the intrinsic location parameters identified between the connected nodes. According to the concept of system of coherent localised entities, if an intrinsic location parameter is recognised to exist between one feature of a system $S_{L o 1}$ and one feature of a system $S_{L o 2}$, an unequivocally identified dimensional parameter can be associated between each feature of $S_{L o 1}$ and each feature of $S_{L o}$. The identification of this single intrinsic location parameter is coherent with satisfying the transitive property.

\section{Application example}

The methodology described in the previous sections has been implemented in an original software, coded in $\mathrm{C}++$, by using a library dedicated to the processing of tessellated geometric models, which has been developed at the University of L'Aquila.

In order to verify the reliability of the proposed methodology, a specific test case has been analysed which refers to a real object whose acquisition has been carried out by means of an optical scanner (www.scansystems.it). The proposed method has been verified in the identification of some qualitative aspects, such as the features identification, the SHPE recognition, the MORG, MLRG and ILPG resulting. The figure 6 shows the results for the features identification and their respective labels. The areas coloured in black are recognised as non - regular and are therefore automatically excluded from the error evaluation. The situation features are all evaluated by using the $\mathrm{L}_{2}$-rule (Di Angelo et al., 2010).

Figure 6. The results of the features identification and their respective labels

Figure 7 shows the results obtained for the $S_{h / /}$ recognition of the object in figure 6 when using the software here implemented:

- the SHPE Report dialogue box (SHPE being an acronym for system of homogeneous parallel entities) reports the total number of $S_{/ / \mathrm{R}}$ and $S_{/ / \mathrm{P}}$ recognised;

- in figure 7b, a SHPE label is assigned to each $S_{h / / .}$ The features belonging to the same $S_{h / /}$ are depicted in the same colour;

- in figures $7 \mathrm{c}$ and $7 \mathrm{~d}$, the features belonging to each $S_{/ / \mathrm{p}}$ and $S_{/ / R}$, respectively, are listed with the corresponding reference spatial direction. 
Figure 7. The results of the SHPE recognition for the test case under examination

Figure 8 displays the report screen and the graph resulting from the recognition of orientation properties for the test case here analysed (the nodes are depicted in the same colour as the corresponding SHPE reported in figure 7). Figure 9, in turn, shows the report screen and the MLRG obtained when analysing the test case being considered (the graph nodes are the same colour as the corresponding features in figure 6). Finally, the resulting $I L P G$ is represented in figure 10. Each arc of the graph is associated with the distance between the corresponding non-ideal features.

The method has been verified also in the evaluation of some quantitative aspects, such as the analytical identification of the situation features and the evaluation of intrinsic characteristics for each recognised non ideal feature. In table 7 the situation feature and the intrinsic parameter related to each feature recognized for the object under examination (fig. 6) are reported. The differences of orientation from ideal condition are due to the geometric errors of the object. The four holes (cy_0, cy_1, cy_2 and cy_3) which are nominally equal, have different values of their intrinsic parameters (the radius of the cylinder). This variability is due to the error in the holes manufacturing. Furthermore, from some intrinsic situation characteristics the related dimension has been evaluated by using two different Virtual Gauges based on different rules (table 8). It is evident that both the value of each distance and the direction of the corresponding Virtual Gauge depend on the rule used to evaluate the Virtual Gauge.

Table 7. The situation features and the intrinsic characteristics of the non - ideal features recognised

Figure 8. The MORG resulting for the test case analysed.

Figure 9. The $M L R G$ resulting for the test case analysed.

Figure 10. The resulting ILPG for the test case analysed.

Table 8 . The effect of the rule in the distance evaluation

\section{Conclusion}

For some years now, our research group has been developing a new methodology for automatic tolerance inspection starting from an acquired highdensity 3D model. In a previous paper (Di Angelo et al. 2010), we proposed a general approach for automatic form error evaluation and specification. That approach was founded on the concepts of non-ideal feature and intrinsic nominal reference. The 
object to be inspected was segmented into a set of non-ideal features, for each of which one or more intrinsic nominal references were identified. Based on those concepts, new and old categories of form errors were defined and some procedures were proposed for a more robust process of verification of traditional tolerance categories.

In order to complete the category of intrinsic nominal references, this paper goes further and introduces new mutual intrinsic orientation, location and dimensional properties pertaining to $3 \mathrm{D}$ features. Moreover, with a view to grouping together all the information recognisable in a scanned object, a new data structure called Recognised Geometric Model (RGM) is presented. The recognition of nominal categories in a scanned object is carried out based on some rules which therefore play a leading role in the definition of the domain of the representable entities within $R G M$. In particular, this paper puts forth the rules concerning the recognition of intrinsic properties in the orientation and location relationships existing between non ideal features. The concept of Virtual Gauge is also introduced and the effect of the rule which has been used to evaluate it is highlighted in the analysis of a real case.

Based on the geometric product specification, the $R G M$ data structure can be queried to gather some quantitative information concerning special intrinsic geometric parameters and/or non-idealities. Future work should address how to specify the tolerated errors in accordance with the $R G M$ data structure and how to measure the non-idealities of the object.

\section{References}

Di Angelo, L., Di Stefano, P., Morabito, A., 2007. Fuzzy sets for geometric shape recognition in triangular meshes. In Proceedings of the 6th International Conference on Intelligent Processing and Manufacturing of Materials (IPMM), Salerno, Italy, $25 \div 29$ June 2007, [CD - ROM Di Angelo Paper1.pdf].

Di Angelo, L., Di Stefano, P., 2010. $C^{1}$ continuities detection in triangular meshes. Computer Aided Design, 42 (9), 828-839.

Di Angelo, L., Di Stefano, P., Morabito, A., 2010. Automatic evaluation of form errors in high-density acquired surfaces. International Journal of Production Research, First published on 26 April 2010.

Gao, J., Gindy, N., Chen, X., 2006. An automated GD\&T inspection system based on non-contact 3D digitization. International Journal of Production Research, 44 (1), 117-134.

Gross, L., Yellen, J., 2004. Handbook of Graph Theory, CRC Press, Boca Roton.

ISO/TS 17450-1: 2005: "Geometrical product specification (GPS) - General concept - Part 1: Model for geometric specification and verification".

ISO/TS 17450-2: 2006: "General concepts - Part 2: Basic tenets, specifications, operators and uncertainties".

Li, Y. and Gu, P., 2004. Free-form surface inspection techniques - state of the art review. Computer-Aided Design, 36 (13), 1395-1417.

Li, Y. and Gu, P., 2005. Inspection of free-form shaped parts. Robotics and Computer Integrated Manufacturing, 21 (4 - 5), 421-430.

Nielsen, H. S., 2006. New Concepts in Specifications, Operators and Uncertainties and Their Impact on Measurement and Instrumentation. Measurement Science and Technology, 17 (3), 541-544. 
Prieto, F., Redarce, T., Lepage R., Boulanger, P., 2002. An Automated Inspection System. International Journal of Advanced Manufactured Technology, 19 (12), 917-925.

Prieto, F., Lepage, R., Boulanger, P., Redarce, T., 2003. A CAD-based 3D data acquisition strategy for inspection. Machine Vision and Applications, 15 (2), 76-91.

Son, S., Park, H., Lee, K. H., 2002. Automated laser scanning system for reverse engineering and inspection. International Journal of Machine Tools \& Manufacture, 42 (8), 889-897.

Srinivasan, V., An Integrated view of Geometrical Product Specification and Verification. In Proceedings of the 7th CIRP Seminar on Computer-Aided Tolerancing, Specification and Verification, 24-25 April 2001, Cachan, France.

Wong F.S.Y., Chuah K.B. and Venuvinod P.K., 2006. Automated inspection process planning: algorithmic inspection feature recognition, and case representation for CBR. Robotics and Computer-Integrated Manufacturing 2 (2006), 56-68. 


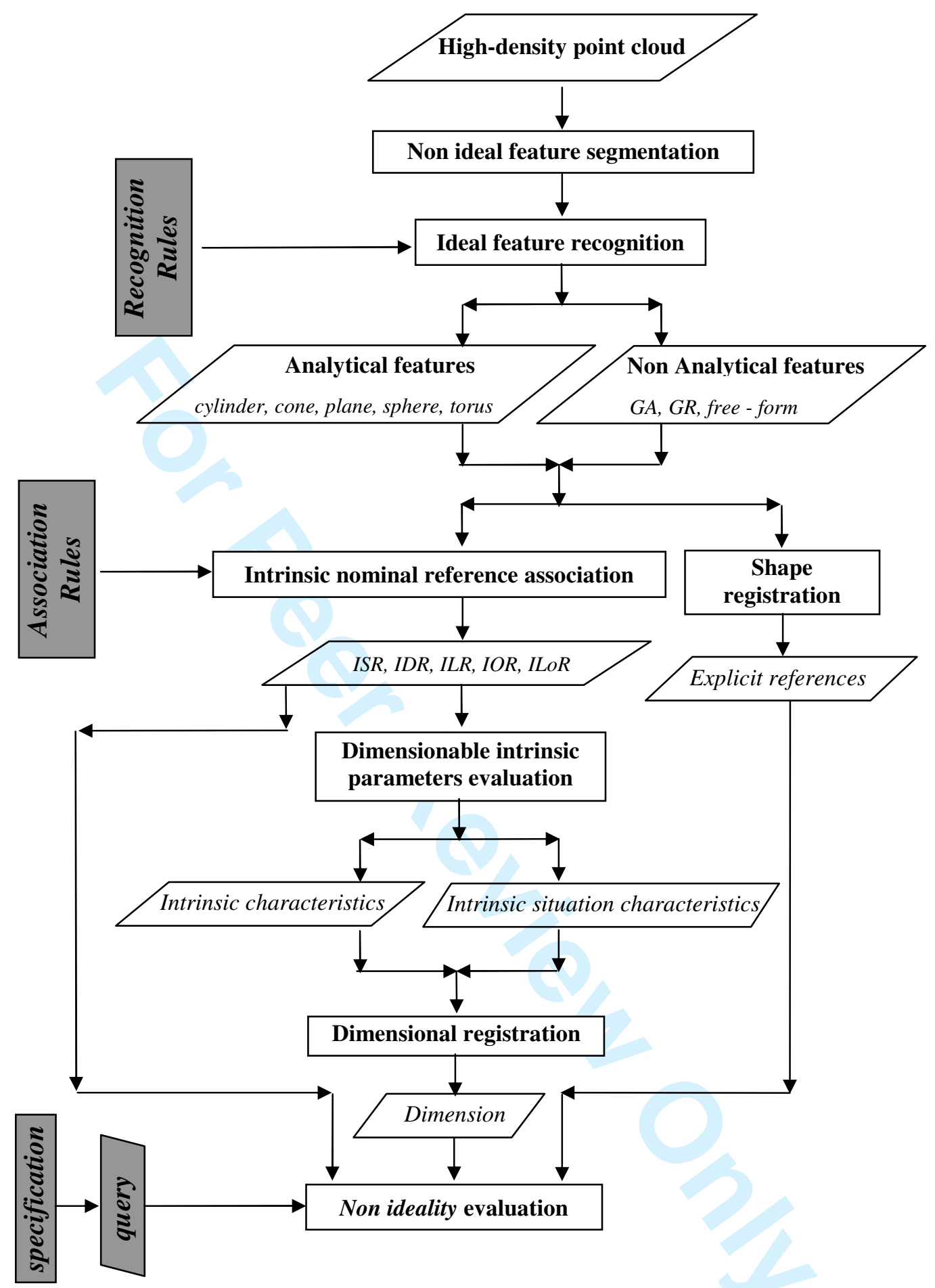

Figure 1: Flowchart for the process of derivation and query of the $R G M$ 
a)
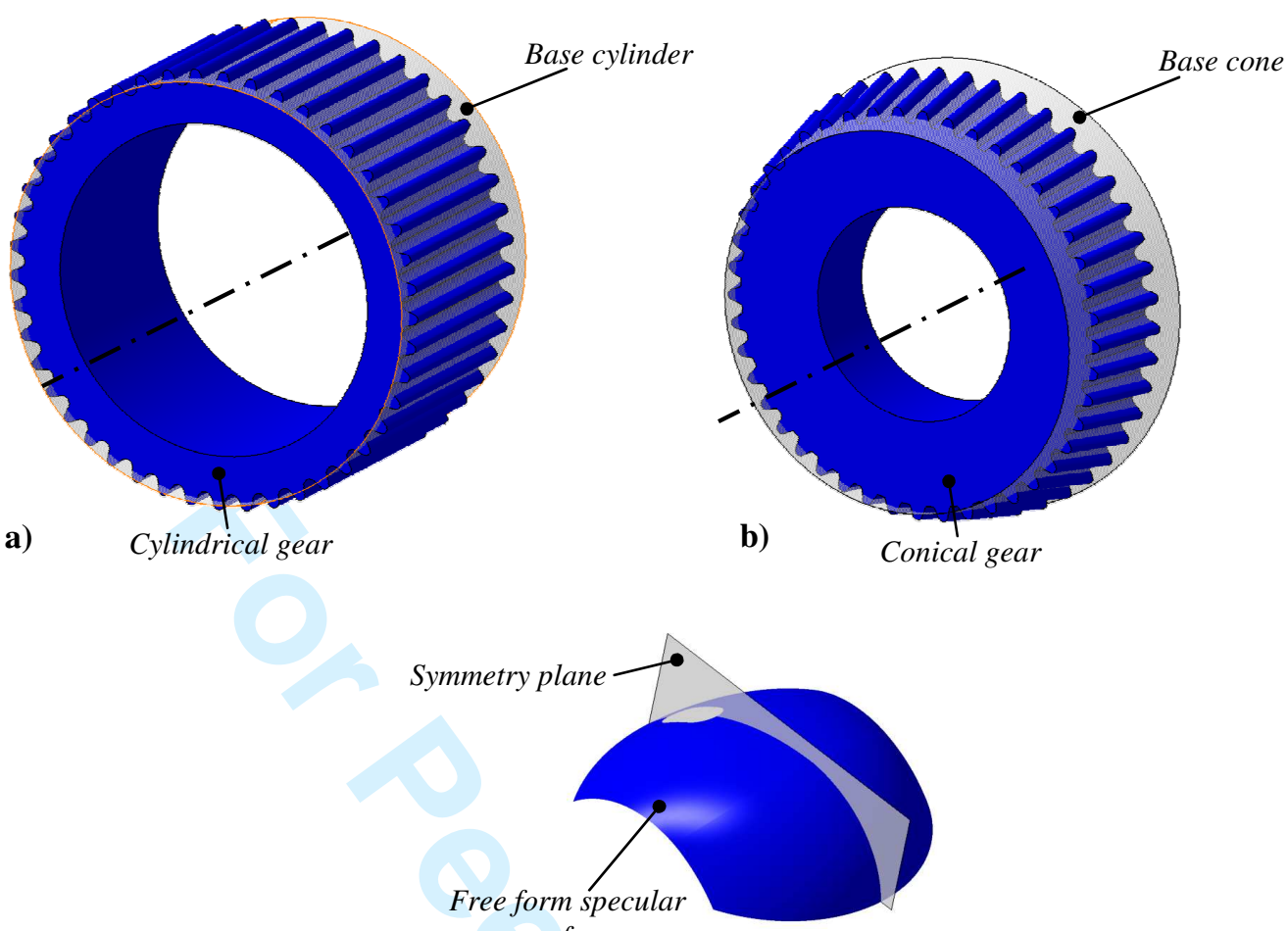

c) surface

Figure 2: Examples of IDRs: a) the base cylinder for a cylindrical gear, b) the base cone for a conical gear and c) the symmetry plane for a free-form specular surface. 
a)



b)


Figure 3: An example of recognition of orientation properties and related graphs 




Figure 4: Derivation of several systems of homogenous parallel entities from one system that is not $S_{h / l}$ 




Figure 5: The three intrinsic location characteristics identified for a system $S_{/ / P}$ of three planar features


Figure 6. The results of the features identification and their respective labels 



Figure 7. The results of the SHPE recognition for the test case under examination 




Figure 8 . The $M O R G$ resulting for the test case analysed.


Figure 9. The $M L R G$ resulting for the test case analysed. 




Figure 10. The resulting ILPG for the test case analysed. 
Table 1. The Intrinsic Characteristics of the ISRs

\begin{tabular}{c|c} 
Type of ISRs & Intrinsic Characteristics \\
\hline Plane & none \\
\hline Sphere & diameter \\
\hline Cylinder & diameter \\
\hline Cone & apex angle
\end{tabular}

Table 2. The situation features for each type of non-ideal feature here considered

\begin{tabular}{l|rl} 
Type of non ideal feature & Situation features \\
\hline Plane & $\circ$ the plane $\Pi ;$ \\
\hline Sphere & $\circ$ the centre $\wp ;$ \\
\hline Cylinder & $\circ$ the axis $r ;$ \\
\hline Cone & $\circ$ the axis $r ;$ \\
& $\circ$ the apex $\wp ;$ \\
\hline Generic axial-symmetric & $\circ$ the axis $r ;$ \\
\hline Generic extruded & $\circ$ the extrusion direction $r ;$ \\
\hline Generic cone & $\circ$ the apex $\wp ;$ \\
\hline Free form & $\circ \quad---------------------$ \\
\hline
\end{tabular}

Table 3. Ideal feature types and the related $I S R \mathrm{~s}, I D R \mathrm{~s}$ and $I L R \mathrm{~s}$

\begin{tabular}{|c|c|c|c|c|c|}
\hline & \multirow{2}{*}{\multicolumn{2}{|c|}{ Ideal feature type }} & \multicolumn{3}{|c|}{ Intrinsic Reference Types } \\
\hline & & & ISR & IDR & $I L R$ \\
\hline \multirow{4}{*}{ 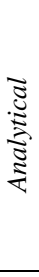 } & \multicolumn{2}{|l|}{ Plane } & Analytical shape & Normal direction- & Surface regularity \\
\hline & \multicolumn{2}{|l|}{ Sphere } & Analytical shape & Centre & Surface regularity \\
\hline & \multicolumn{2}{|l|}{ Cylinder } & Analytical shape & $\begin{array}{l}\text { Axis } \\
\text { Ideal circumferential line }\end{array}$ & $\begin{array}{c}\text { Surface regularity } \\
\text { Profile regularity } \\
\text { Ruledness }\end{array}$ \\
\hline & \multicolumn{2}{|l|}{ Cone } & Analytical shape & $\begin{array}{c}\text { Axis } \\
\text { Ideal circumferential line }\end{array}$ & $\begin{array}{c}\text { Surface regularity } \\
\text { Profile regularity } \\
\text { Ruledness }\end{array}$ \\
\hline \multirow{4}{*}{  } & \multirow{2}{*}{$\begin{array}{l}\text { Generic Ruled } \\
\qquad(G R)\end{array}$} & generic extruded & & Extrusion Direction & $\begin{array}{c}\text { Surface regularity } \\
\text { Profile regularity } \\
\text { Ruledness }\end{array}$ \\
\hline & & generic cone & & Apex vertex & $\begin{array}{c}\text { Surface regularity } \\
\text { Ruledness }\end{array}$ \\
\hline & \multicolumn{2}{|c|}{ Generic Axially - symmetric (GA) } & - & $\begin{array}{l}\text { Axis } \\
\text { Ideal circumferential line }\end{array}$ & $\begin{array}{l}\text { Surface regularity } \\
\text { Profile regularity }\end{array}$ \\
\hline & \multicolumn{2}{|c|}{ Free form } & - & & Surface regularity \\
\hline
\end{tabular}

Table 4. The rules to recognise the intrinsic orientation properties

\begin{tabular}{|c|c|c|}
\hline \multicolumn{2}{|c|}{ dot product value } & \multirow[b]{2}{*}{ Intrinsic Orientation Property } \\
\hline $\begin{array}{c}\text { Features of the } \\
\text { same type }\end{array}$ & $\begin{array}{l}\text { Features of a } \\
\text { different type }\end{array}$ & \\
\hline 0 & 1 & perpendicularity \\
\hline \pm 0.5 & $\pm \sqrt{3} / 2$ & angular orientation of $60^{\circ},-60^{\circ}, 120^{\circ}$ and $240^{\circ}$ \\
\hline$\pm \sqrt{2} / 2$ & $\pm \sqrt{2} / 2$ & angular orientation of $45^{\circ},-45^{\circ}, 135^{\circ}$ and $225^{\circ}$ \\
\hline$\pm \sqrt{3} / 2$ & \pm 0.5 & angular orientation of $30^{\circ},-30^{\circ}, 150^{\circ}$ and $210^{\circ}$ \\
\hline \pm 1 & 0 & parallelism \\
\hline
\end{tabular}


Table 6. The coincidence property recognition

Table 5. The intrinsic location properties here recognised for the several feature types

\begin{tabular}{c|c|c|c|c|c} 
Feature type & Plane & Sphere & Cylinder & Cone & $\begin{array}{c}\text { Generic Axially - symmetric } \\
\text { (GA) }\end{array}$ \\
\hline Plane & Coincidence & - & - & - & - \\
\hline Sphere & - & $\begin{array}{c}\text { Concentricity } \\
\text { Coincidence }\end{array}$ & - & - & Coaxiality \\
\hline Cylinder & - & - & $\begin{array}{c}\text { Coaxiality } \\
\text { Coincidence }\end{array}$ & Coaxiality & Coaxiality \\
\hline Cone & - & - & Coaxiality & $\begin{array}{c}\text { Coaxiality } \\
\text { Coincidence }\end{array}$ & Coaxiality
\end{tabular}

Table 7. The situation features and the intrinsic characteristics of the non - ideal features recognised

\begin{tabular}{|c|c|c|c|c|c|c|c|}
\hline \multirow{3}{*}{$\begin{array}{c}\text { Feature } \\
\text { name }\end{array}$} & \multicolumn{6}{|c|}{ Situation features } & \multirow{3}{*}{$\begin{array}{c}\text { Intrinsic } \\
\text { parameters } \\
\text { Radius [mm] }\end{array}$} \\
\hline & \multicolumn{3}{|c|}{ Base point [mm] } & \multicolumn{3}{|c|}{ Direction } & \\
\hline & $\mathbf{x}$ & $\mathbf{y}$ & $\mathbf{z}$ & $\mathbf{x}$ & $\mathbf{y}$ & $\mathbf{z}$ & \\
\hline cy_0 & 25.44 & 82.27 & 5.36 & 0.009 & 0.041 & 0.999 & 11.05 \\
\hline cy_1 & -25.76 & 82.35 & 8.16 & 0.009 & 0.003 & 0.999 & 10.83 \\
\hline cy_2 & -25.41 & -81.18 & 1.15 & 0.004 & -0.001 & -0.999 & 11.13 \\
\hline cy_3 & 25.81 & -81.21 & 6.81 & 0.013 & 0.015 & -0.999 & 11.03 \\
\hline cy_4 & -0.067 & 0.23 & -0.42 & -0.001 & 0.002 & 0.999 & 31.66 \\
\hline pl_5 & 50.38 & 1.07 & 17.07 & 0.999 & -0.001 & -0.001 & -- \\
\hline pl_6 & 0.41 & 2.01 & 0.09 & 0.001 & 0.000 & -1 & -- \\
\hline pl_7 & -0.19 & -0.51 & 40.20 & 0.002 & -0.001 & 0.999 & -- \\
\hline pl_8 & -0.27 & -80.59 & 19.67 & -0.001 & -0.000 & 1 & -- \\
\hline pl_9 & 0.06 & 80.93 & 19.70 & -0.000 & 0.000 & 1 & -- \\
\hline pl_10 & -50.36 & -1.00 & 16.97 & -1.0 & -0.002 & -0.002 & -- \\
\hline pl_11 & -0.88 & 50.52 & 29.84 & 0.002 & 0.999 & -0.013 & -- \\
\hline pl_12 & 0.42 & -0.42 & 14.56 & 0.002 & -0.001 & 0.999 & -- \\
\hline pl_13 & -0.14 & -50.26 & 29.83 & -0.002 & $\begin{array}{l}-0.999 \\
\end{array}$ & -0.027 & -- \\
\hline pl_14 & 0.05 & 111.91 & 9.69 & 0.002 & 0.999 & 0.007 & -- \\
\hline pl_15 & 0.07 & -111.09 & 10.23 & 0.002 & -1 & 0.001 & -- \\
\hline pl_16 & 45.14 & 106.66 & 9.85 & 0.707 & 0.707 & 0.013 & -- \\
\hline pl_17 & -45.12 & 106.64 & 10.15 & -0.707 & 0.707 & 0.003 & -- \\
\hline pl_18 & 45.02 & -105.88 & 10.04 & 0.707 & -0.707 & 0.004 & -- \\
\hline pl_19 & -45.13 & -105.77 & 10.13 & -0.706 & -0.708 & -0.001 & -- \\
\hline cy_20 & 0.28 & -0.35 & -143.34 & -0.003 & 0.004 & 0.999 & 21.24 \\
\hline
\end{tabular}


Table 8 . The effect of the rule in the distance evaluation

\begin{tabular}{|c|c|c|c|c|c|c|c|c|c|}
\hline \multirow{3}{*}{\multicolumn{2}{|c|}{$\begin{array}{l}\text { Parallel } \\
\text { features }\end{array}$}} & \multicolumn{4}{|c|}{$\mathrm{L}_{1}$-rule } & \multicolumn{4}{|c|}{$\mathbf{L}_{2}$-rule } \\
\hline & & \multicolumn{3}{|c|}{ Virtual Gauge direction } & \multirow{2}{*}{$\begin{array}{c}\text { distance } \\
{[\mathrm{mm}]}\end{array}$} & \multicolumn{3}{|c|}{ Virtual Gauge direction } & \multirow{2}{*}{$\begin{array}{c}\text { distance } \\
{[\mathrm{mm}]}\end{array}$} \\
\hline & & $\mathbf{x}$ & $\mathbf{y}$ & $\mathbf{z}$ & & $\mathbf{x}$ & $\mathbf{y}$ & $\mathbf{z}$ & \\
\hline pl_10 & cy_2 & $1.36 \cdot 10^{-4}$ & $-1.23 \cdot 10^{-4}$ & 1.000 & 24.38 & $2.71 \cdot 10^{-4}$ & $-1.59 \cdot 10^{-4}$ & 1.000 & 24.67 \\
\hline pl_10 & cy_3 & $5.34 \cdot 10^{-3}$ & 0.03811 & 0.999 & 76 & 0.010 & 0.042 & 0.999 & 76.29 \\
\hline pl_10 & pl_5 & 0.999 & $7.4 \cdot 10^{-4}$ & $-1.32 \cdot 10^{-4}$ & 100.92 & 1. & $2.9 \cdot 10^{-5}$ & $-8.11 \cdot 10^{-4}$ & 100.98 \\
\hline cy_2 & cy_3 & $-8.2 \cdot 10^{-5}$ & $-2.53 \cdot 10^{-3}$ & -0.999 & 51.31 & $-3.6 \cdot 10^{-3}$ & -0.016 & -0.999 & 51.28 \\
\hline cy_2 & pl_5 & $-1.26 \cdot 10^{-3}$ & $-1.60 \cdot 10^{-3}$ & -0.999 & 75.87 & $-2.13 \cdot 10^{-4}$ & $-3.42 \cdot 10^{-3}$ & -0.999 & 75.98 \\
\hline cy_3 & pl_5 & $-1.26 \cdot 10^{-3}$ & $-1.60 \cdot 10^{-3}$ & -0.999 & 24.06 & $2.78 \cdot 10^{-3}$ & 0.010 & -0.999 & 24.38 \\
\hline
\end{tabular}

